\title{
Multiversal SpaceTime (MSpaceTime) Not Neural Network as Source of Intelligence in Generalized Quantum Mechanics, Extended General Relativity, Darwin Dynamics for Artificial Super Intelligence Synthesis
}

\author{
Yang Zhang \\ Multiverse Corp \\ hwswworld@yandex.com
}

This article has been withdrawn by bioRxiv owing to a screening error. 